\title{
1 Social isolation modulates appetite and defensive behavior via a common \\ 2 oxytocinergic circuit in larval zebrafish
}

4 Caroline L. Wee ${ }^{1,2,3 \S}$, Erin Song ${ }^{1 \S}$, Maxim Nikitchenko ${ }^{1,4 \S}$, Sandy Wong ${ }^{1}$, Florian Engert $^{1 *}$,

5 Samuel Kunes ${ }^{1 *}$

7 ङEqual-contribution first authors

$8 \quad{ }^{*}$ Corresponding authors

Affiliations:

11 Department of Molecular and Cellular Biology and Center for Brain Science, Harvard

12 University, Cambridge, Massachusetts, USA

$13{ }^{2}$ Program in Neuroscience, Department of Neurobiology, Harvard Medical School, Boston,

14 Massachusetts, USA

$15{ }^{3}$ Present address: Institute of Molecular and Cell Biology, $A^{*}$ STAR, Singapore

$16 \quad{ }^{4}$ Present address: Duke University, Durham, North Carolina, USA

\section{Correspondence:}

19 Florian Engert / Samuel Kunes

20 Department of Molecular and Cellular Biology

21 Harvard University, 16 Divinity Ave, Biolabs 2073

22 Cambridge, MA 02138, USA

\section{ABSTRACT}

25 How brains encode social stimuli and transform these representations into advantageous behavioral responses is not well-understood. Here, we show that social isolation activates an oxytocinergic, nociceptive circuit in the larval zebrafish hypothalamus. We further demonstrate that chemical cues released from conspecific animals modulate its activity to regulate defensive behaviors and appetite. Our collective data reveals a model through which social stimuli can be integrated into fundamental neural circuits to mediate adaptive behaviour. 


\section{INTRODUCTION}

In mammals, signaling in oxytocinergic (OXT) circuits modulates a wide spectrum of socially driven behaviors, ranging from pair bonding and parental care to the responses to stress and pain $^{1-3}$. OXT has also been described as a potent regulator of appetite ${ }^{4,5}$. We reported recently that the larval zebrafish OXT circuit encodes a response to aversive, particularly noxious stimuli and directly drives nocifensive behavior via brainstem premotor targets ${ }^{6}$. Moreover, studies in both zebrafish $^{6-8}$ and mammals ${ }^{9,10}$ suggest that the OXT-expressing neuronal population is anatomically and functionally diverse, and might also modulate multiple behaviors in zebrafish. Here, in a brain-wide screen ${ }^{11}$ for neuronal populations whose activity reflects social context, we show that larval zebrafish oxytocinergic circuits display diverse responses to conspecific chemosensory stimuli, and are key effectors for social context modulation of nociceptive and appetite-driven behaviors. Our results reveal a simple algorithm by which neuromodulatory neurons can represent social context to exert flexible control over hard-wired behavioral drives.

\section{RESULTS}

Brain-wide activity mapping of social isolation and its rescue by conspecific chemical cues Using pERK based whole-brain activity mapping (MAP-Mapping ${ }^{11}$ ), neural activity in brains of briefly ( 2 hrs) socially-isolated larvae (7 - 8 days-post-fertilization; dpf) was compared to animals that had been maintained in the presence of similarly-aged conspecifics. We found that isolated fish showed an enhancement of neural activity in specific regions, including the telencephalon (especially subpallium), hindbrain, locus coeruleus, area postrema, caudal hypothalamus, preoptic area (PO, homolog of the hypothalamic paraventricular nucleus in mammals) and posterior tuberculum (PT) (Fig. 1a-b, Supplementary Movie 1, Supplementary Data 1). Many of these same regions are activated by noxious or aversive stimuli ${ }^{6,7}$; they may thus represent the signature activity pattern of a negative internal state, which can be similarly triggered by social deprivation. Neurons expressing the peptide oxytocin (OXT) are abundant in the $\mathrm{PO}$ and $\mathrm{PT}^{6,12,13}$ regions, and as we describe below, OXT-positive neuron clusters in both of these areas (OXT $T_{P O}$ and $O X T_{P T}$ respectively) display greater activity in socially-isolated fish. To acquire a more precise quantitation of OXT activity in relation to the social environment, we measured pERK activity for individual GFP-labeled OXT neurons ( $T g(o x t: G F P)$ ), as well as surrounding non-OXT PO and PT neurons, in high resolution confocal microscopic images of dissected brains $^{6}$ (Fig. 1c-d, Supplementary Fig. 1). We found that a subset of both OXT neurons and surrounding non-OXT neurons were significantly more active in socially-isolated 


\section{FIGURE 1}

a

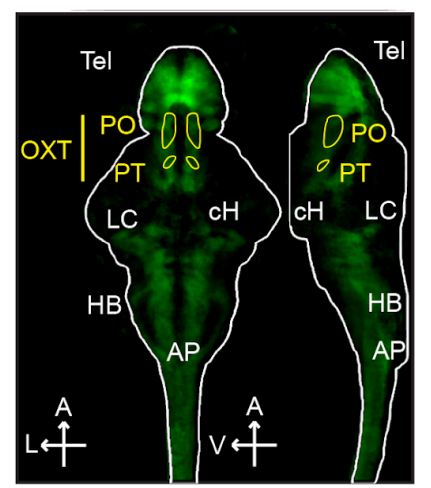

Isolated (2 hrs) > Group

- Isolated (2 hrs) < Group (not detected)

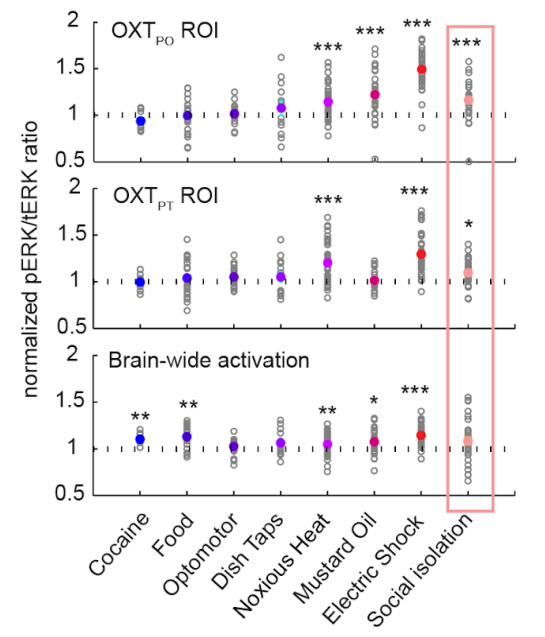

C anti-pERK staining (OXT neurons, averaged by fish)

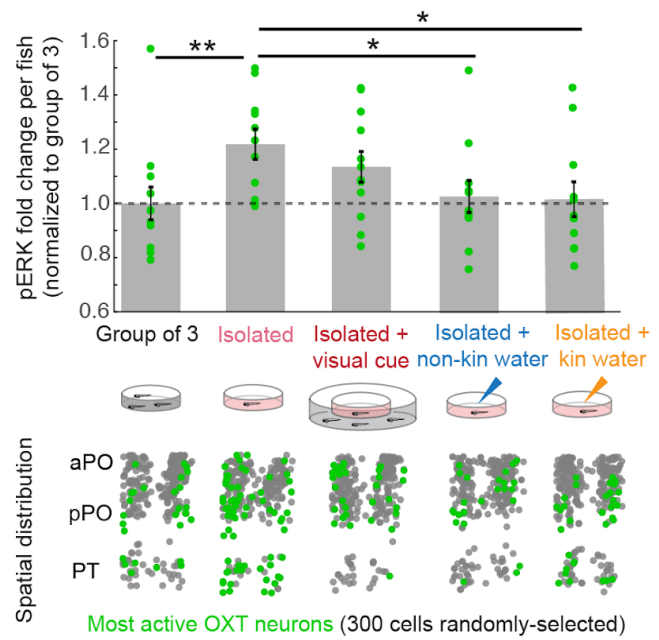

b Group of 3

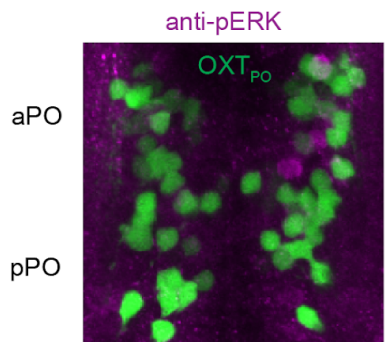

PT
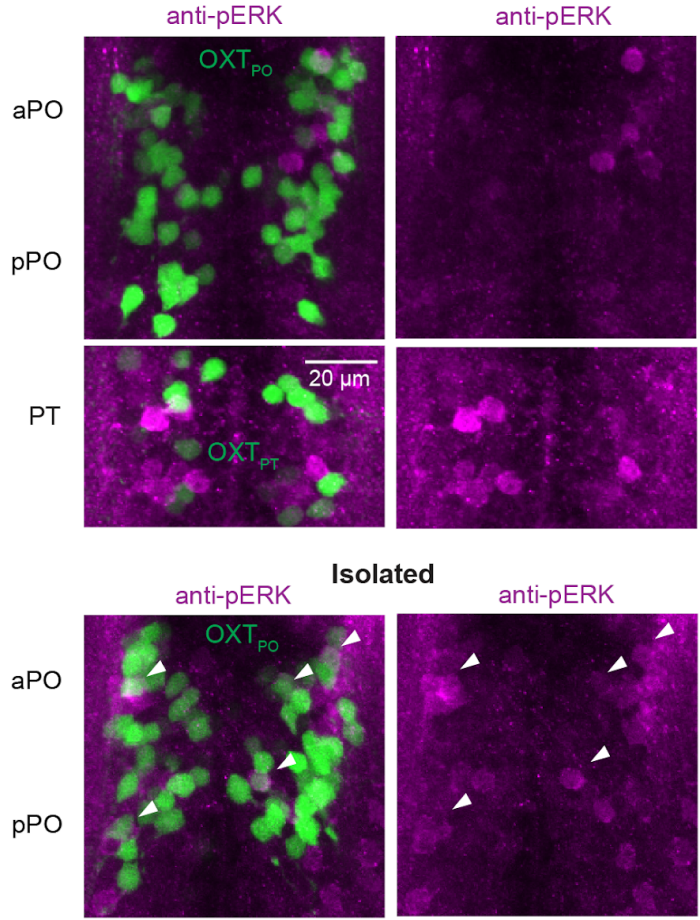

PT
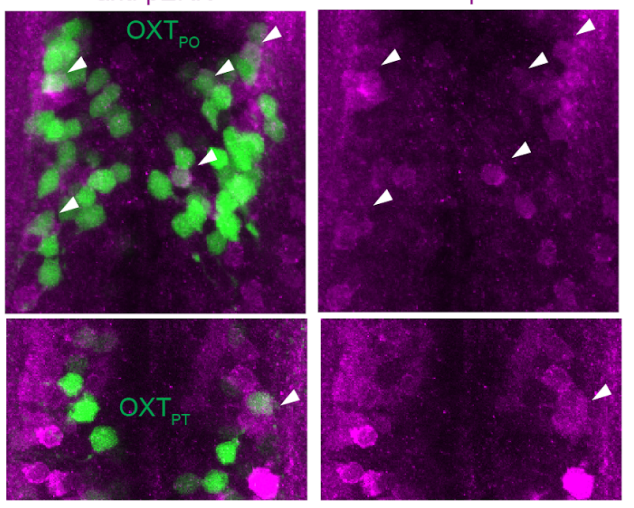

d anti-pERK staining (OXT neurons, pooled across all fish)

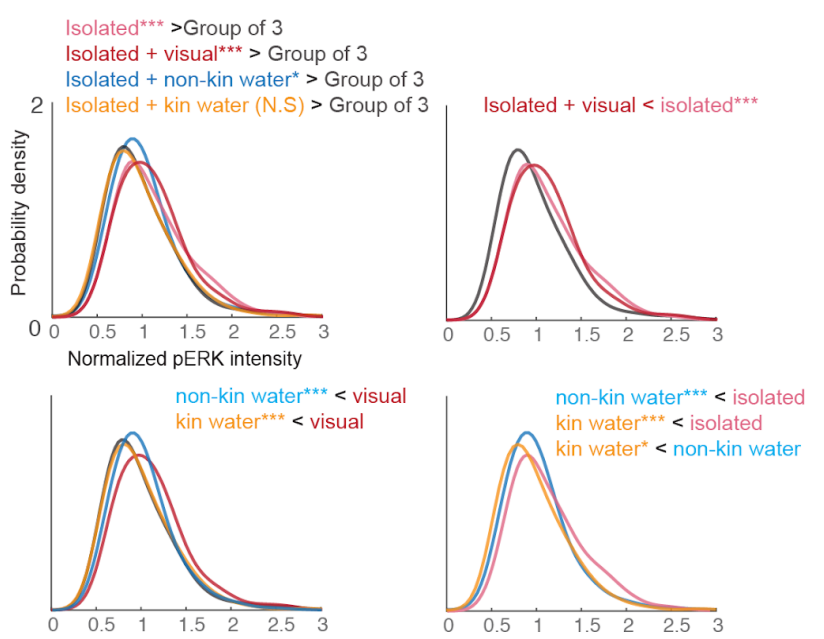


Figure 1: phospho-ERK based mapping reveals OXT neuron modulation by social context

(a) Top: pERK-based activity mapping to compare brain activity of isolated vs. social fish. Green voxels highlight regions that are significantly more active in isolated fish as compared to fish maintained in groups. This final map was generated by averaging data from 5 independent experiments in which isolated fish are compared to fish kept in groups of 3 (2 experiments), 5 (1 experiment) or 10 (2 experiments) (See Supplementary Movie 1; FDR Threshold used $=0.05 \%$. Yellow $=$ Outline of preoptic $\left(\mathrm{OXT}_{\mathrm{PO}}\right)$ and posterior $\left(\mathrm{OXT}_{\mathrm{PT}}\right)$ oxytocin populations). These masks were used for the quantification of activity in bottom. Other ROls are quantified in Supplementary Data 1. Tel $=$ Telencephalon, $\mathrm{cH}=$ Caudal Hypothalamus, LC = Locus Coeruleus, AP = Area Postrema, HB = Hindbrain.

Bottom: Social isolation significantly activates OXT-expressing brain regions. Here, the mean $p E R K$ signal was calculated per fish across the specified ROls. Data for other stimuli was also included in Wee

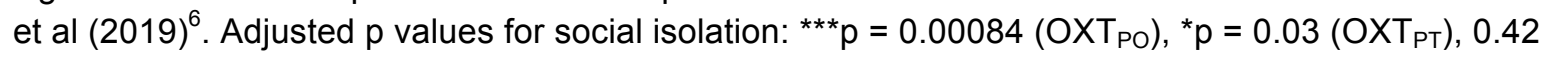
(whole brain). Wilcoxon signed-rank test relative to a median of 1 , Bonferroni correction.

(b) High-resolution imaging of pERK expression in OXT neurons in brains from dissected isolated fish (bottom), compared to the brains of fish kept in groups of 3 (top). Maximum intensity projection of one fish from each condition are shown (green = preoptic $\left(O X T_{P O}\right)$ and posterior $\left(O X T_{P T}\right)$ oxt:GFP-positive neurons, magenta $=$ anti-pERK staining). White arrows indicate OXT neurons with high pERK intensities. Scale bar $=20 \mu \mathrm{m}$.

(c) Top: Effect of social isolation and social sensory cues on mean OXT (PO + PT combined, green) neuron activity per fish. pERK intensities of individual OXT and non-OXT (see Supplementary Fig. 1) cells were extracted automatedly as reported in Wee et al $(2019)^{6}$. pERK intensities shown are normalized to those of control fish (i.e. fish maintained in groups of 3). Social isolation induced a 1.2 fold increase in OXT neuron pERK activity relative to fish maintained in groups of 3 . Visual cues of conspecifics moderately reduced OXT neuron pERK activity ( 1.1 fold relative to group), non-kin and kin water cues induced an even stronger reduction in OXT neuron pERK activity (1.01 and 1.00 fold relative to group). OXT neurons: $p=0.0074^{* *}$ ( group vs isolated) / 0.069 (group vs visual) / 0.52 (group vs nonkin water) / 0.83 (group vs kin water) / 0.37 (isolated vs visual) / $0.022^{*}$ (isolated vs non-kin water) / $0.036^{*}$ (isolated vs kin water) / 0.21 (visual vs non-kin water) / 0.19 (visual vs kin water) / 0.65 (kin vs non-kin water). Bottom: Spatial distribution of the most active OXT-positive neurons. Active neurons were defined using a pERK intensity threshold above which only $10 \%$ of neurons are active in the control (i.e group of 3 ) condition. 300 OXT cells were randomly sampled across all fish.

(d) Probability distributions (kernel density estimate (KDE)) of normalized pERK fluorescence across all neurons per group. Multiple panels are shown to aid visualization. Fish were either kept in groups of 3 (gray, $\mathrm{n}=993$ neurons from 12 fish), isolated (pink, $\mathrm{n}=931$ neurons from 11 fish), isolated but exposed to visual cues of conspecifics through a transparent barrier (red, $n=902$ neurons from 12 fish) or isolated but exposed to non-kin-conditioned water (blue, $\mathrm{n}=866$ neurons from 11 fish) or kin-conditioned water (orange, $n=856$ neurons from 12 fish). Isolated fish had significantly higher OXT neuron $\mathrm{pERK}$ activation than fish in a group $\left.{ }^{* * *} p=2.6 \times 10^{-35}\right)$. Isolated fish presented with visual conspecific or non-kin water cues also had significantly higher OXT pERK activity than fish in a group $\left({ }^{* *} p=4.8 \times 10^{-15}\right.$ and ${ }^{*} p=0.027$ respectively), whereas kin water did not significantly change OXT neuron activity relative to fish in group $(p=0.67)$. All cue types significantly reduced OXT neuron activity relative to isolated fish $\left({ }^{* * *} 2.8 \times 10^{-6}\right.$ (visual), ${ }^{* * *} 6.3 \times 10^{-26}$ (non-kin), $\left.{ }^{* * *} 2.0 \times 10^{-34}(\mathrm{kin})\right)$. Kin water induced significantly-lower OXT neuron activity relative to non-kin water $\left({ }^{*} p=0.012\right)$. Both kin and non-kin water reduced OXT neuron activity significantly more than visual cues $\left({ }^{* * *} p=2.9 \times 10^{-15}\right.$ and ${ }^{* * *} p=2.6 \times 10^{-9}$ respectively), two-sided Wilcoxon rank-sum test. 
As a step toward elucidating the nature of the social signal, we compared OXT neuronal activity in animals exposed to the separated visual or chemical cues of a social environment. We find that water conditioned by prior exposure to conspecific larval fish (see Methods) reduced the elevated OXT neuronal activity observed in socially isolated fish, whereas visual exposure to conspecific larval fish (maintained in a separate water enclosure) had minimal effect (Fig. 1c-d). We also examined the effect of water conditioned with similarly-aged sibling (kin) fish in relation to similarly-aged fish of a different strain background (non-kin). Both 'kin' and 'non-kin' conditioned water was sufficient to reduce OXT neuron activity (Fig. 1c-d), but the effect of kin water was significantly stronger (Fig. 1d). Nearby OXT-negative neurons in the PO and PT area were also suppressed by conspecific-conditioned cues (Supplementary Fig. 1). Thus OXT and PO/PT neural activity in larval zebrafish is increased during brief ( $2 \mathrm{hr}$ ) social isolation, and this activity is equivalently (and rapidly, see Fig. 2) suppressed by exposure to water that had held conspecific larval fish.

In vivo calcium imaging reveals diverse olfactory and OXT neuronal responses to chemical

\section{conspecific cues}

139 We next turned to in vivo calcium imaging to acquire a temporally precise record of OXT neuronal activity. Imaging was performed on larvae in which UAS:GCaMP6s was driven directly in OXT neurons with an oxt:Gal4 driver (Fig. 2a). Tethered 8-11 dpf fish were subjected to either conditioned or control water released in 10 second pulses. We show that pure water flow, which represents a mechanosensory stimulus, mildly activated OXT neurons, whereas water that had been conditioned by prior incubation with larval fish triggered an immediate relative reduction of OXT neuronal activity (Fig. 2a,e-f). We next tested whether OXT neuron calcium activity also discriminates between kin and non-kin conspecific cues ${ }^{14,15}$. To that end, OXT neuronal activity was compared between sibling-conditioned water (kin water') and water conditioned by larvae of a distinct genetic background ${ }^{16}$ ('non-kin water'). We also examined water conditioned by the presence of adult kin, which is potentially an aversive predator cue, as adult zebrafish consume their own young. To minimize the effects of familiarity, conspecifics used to generate conditioned cues were raised apart (in a different dish) from experimental fish.

Since water-borne chemical cues likely act as odorants, we included the olfactory bulb

$153(\mathrm{OB})$ in the areas specifically interrogated for changes in neuronal activity upon exposure to 154 these cues. When examined via labeling with the pan-neuronal GCaMP line HuC:GCaMP6s 155 (Fig. 2a-d, Supplementary Fig. 2), each of the three conditioned water types (kin, non-kin and 156 adult kin) generated overlapping but distinct activity signatures within the OB and OXT 
populations (Fig. 2). As expected, kin water led to a relative reduction of the OXT neuron response (Fig. 2e-f). Adult water, on the other hand, led to a net increase in OXT activity with longer lasting dynamics, correlating with similarly longer lasting olfactory responses (Fig. 2c, ef). Notably, non-kin water was less effective in reducing OXT neuron activity than kin water, and it elicited much weaker OB activity (Fig. 2 f, also compare Fig. $2 b$ to $2 e$ ), suggesting some subtle differences between kin and non-kin water effects that had also been observed with $\mathrm{pERK}$ 163 imaging (compare with Fig. 1d).

FIGURE 2
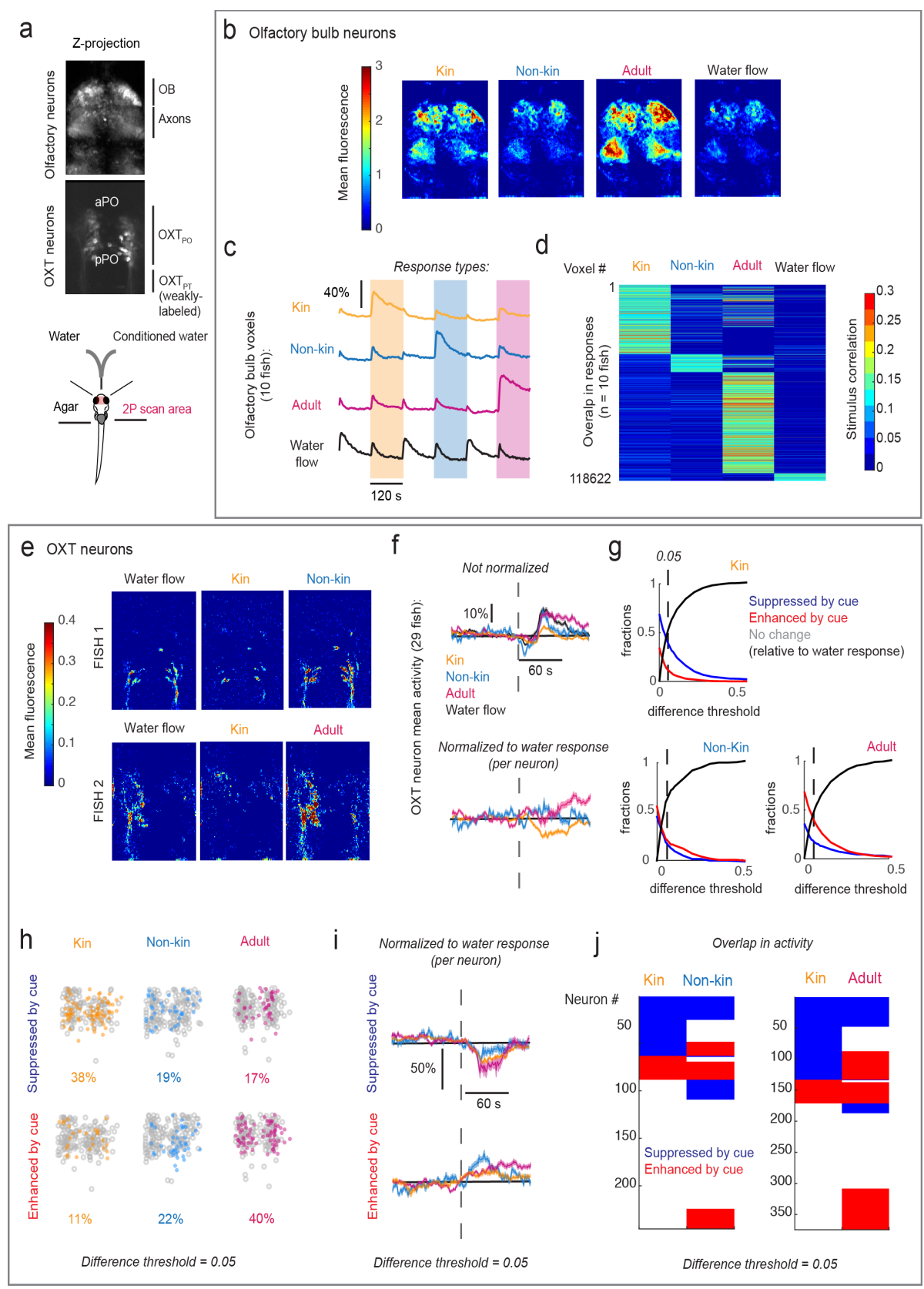
(a) Top: Z-projection of volumetrically-scanned brain regions (olfactory bulb and oxytocin neurons). The olfactory bulb was labeled using $T g$ (HuC:GCaMP6s). The Tg(oxt:Gal4; UAS:GCaMP6s) transgenic line labels OXT $T_{P O}$ neurons strongly, with weaker labeling of the OXT simultaneously. Bottom: Schematic of imaging and cue delivery setup.

\section{(b-d) Olfactory Bulb (OB) Imaging}

(b) Mean $\mathrm{OB}$ fluorescence in response to different stimuli (kin, non-kin, adult or water flow) integrated over a $60 \mathrm{~s}$ period post-stimulus from a single fish. See Supplementary Fig. 2 for more examples.

(c) Mean stimulus-triggered calcium responses from 10 fish. All four stimuli (kin, non-kin, adult or water selective for one of the four stimuli. On average, about $5 / 1.5 / 10 / 1 \%$ of voxels ( $n=17856 / 5357 / 34672 / 3522$ out of 345688 total voxels) were specific to kin, non-kin, adult conditioned water and water respectively. Olfactory responses are sustained and decay slowly throughout the imaging interval (2 min).

(d) Quantification of overlap of OB responses to different stimuli (118622 voxels that are responsive $(r>0.1)$ to at least one of the stimuli, aggregated across 10 fish). While many voxels showed specific activation by individual stimuli, there were some overlapping responses between the cues. Color map indicates Pearson's correlation coefficient ( $r$-value).

\section{(e-j) OXT neuron imaging}

(e) Mean OXT neuron fluorescence integrated over a $60 \mathrm{~s}$ period post-stimulus from two different fish, one imaged with kin and non-kin water, and another with kin and adult water.

(f) Top: Mean stimulus-triggered calcium responses of OXT neurons. All fish ( 29 fish, $n=1033$ neurons) were imaged with water or kin water, some additionally were imaged with either non-kin (8 fish, $n=245$ neurons) or adult water (10 fish, $n=374$ neurons). Bottom: Mean stimulus-triggered calcium responses of each neuron normalized to its own mean water response. Gray broken line indicates stimulus onset. Shading indicates SEM.

(g) Fraction of OXT neurons that would be classified as suppressed (blue) or activated (red) by each water-borne cue, as a function of the mean difference in integrated calcium activity from the water response (i.e. difference threshold). A threshold of 0.05 (i.e. $5 \%$ difference) was used in subsequent panels.

(h) Spatial distribution and percentages of neurons that show either suppressed (top) or enhanced (bottom) responses to each cue relative to water. For accurate comparison of spatial distribution, 200 neurons were randomly selected from each group. Percentages are calculated based on a difference threshold of 0.05 . Kin cues induced the highest percentage suppression (38\%) and lowest percentage activation (11\%), whereas adult cues induced the lowest percentage suppression $(17 \%)$ and highest percentage activation (40\%) of OXT neurons.

(i) Mean stimulus-triggered calcium responses of neurons classified as being suppressed (top) or enhanced (bottom) by each cue, normalized to their mean water response. Shading indicates SEM. 
simultaneously. Red = enhancement, Blue = suppression, White $=$ no change, based on difference threshold of 0.05 . Approximately one third of neurons suppressed by kin water were also suppressed by non-kin $(40 \%)$ and by adult water $(36 \%)$. In addition, a common subset of neurons activated by kin water were also activated by non-kin (76\%) and adult (90\%) water. However, $24 \%$ and $35 \%$ of kin watersuppressed neurons were alternatively activated by non-kin and adult-conditioned water, respectively.

OXT neurons are diverse in their circuit connectivity ${ }^{8,17-19}$ and responses to social deprivation (Fig. 1) and nociceptive input ${ }^{6}$. To further resolve this heterogeneity, we used calcium imaging to classify individual OXT neurons into populations that either reduce or increase their activity in response to water-borne conspecific cues. We found that, across a range of thresholds, the fraction of OXT-positive neurons whose activities were suppressed by larval kin-conditioned water was consistently higher than the fraction of neurons that were activated (Fig. 2g). Non-kin-conditioned water, in contrast, induced equivalent neuronal fractions with increased or suppressed activities. Water conditioned by adult fish triggered a greater fraction of neurons with enhanced activity (Fig. $2 \mathrm{~g}$ ). Under all conditions, activated or suppressed neurons were spatially distributed throughout the OXT-positive PO and PT domains, instead of being segregated into distinct areas (Fig. 2h-i). Further, the specific response properties of individual neurons to one conspecific cue did not predict strongly how they would respond to alternate cues, although we observed that kin water-activated OXT neurons tended to also be commonly activated by other conspecific (non-kin or adult) cues (Fig $2 \mathrm{j}$ ). The heterogeneity of the population suggests that OXT neurons as a group are able to differentially encode these stimuli and provide a basis to discriminate between them in affecting behavior.

Conspecific cues suppress nociceptive OXT circuits and defensive behavior

241 Social buffering is a widely-observed phenomenon in which the presence of conspecifics ameliorates the effects of aversive experience ${ }^{20,21}$. We previously showed that a large fraction of OXT neurons are activated by noxious stimuli and drive defensive behaviors, specifically through brainstem premotor targets that trigger vigorous large-angle tail bends ${ }^{6}$. Given that most of the OXT circuitry appears to be suppressed by water-borne social cues derived from closelyrelated conspecifics, we posited that these cues might reduce the nocifensive behavior induced by the stimulation of TRPA1 receptors. Indeed, increased swim speed triggered by nociceptive

248 TRPA1 receptor activation was significantly ameliorated by the presence of kin-conditioned water (Supplementary Fig. 3). Consistent with the idea that this reduced nocifensive response involves the suppression of OXT neurons, kin water significantly also reduced the peak tail 
a

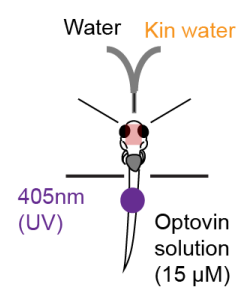

Alternating

Water or kin water

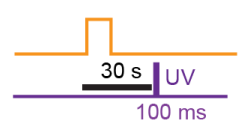

d Activity suppressed by kin water Activity enhanced by kin water
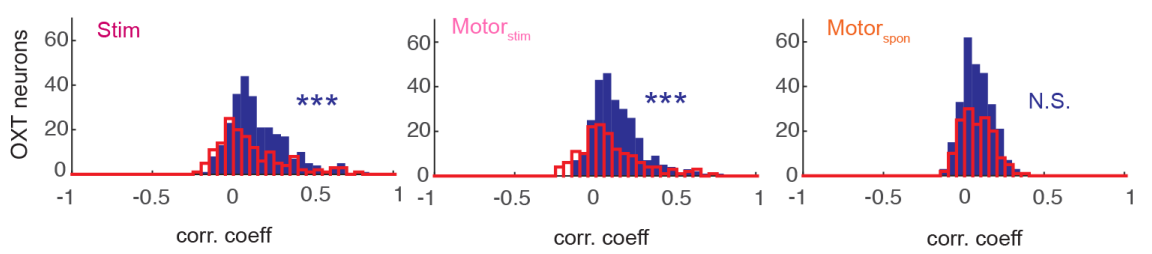

b Tethered behavior kinematics $(\mathrm{n}=16 \mathrm{fish})$
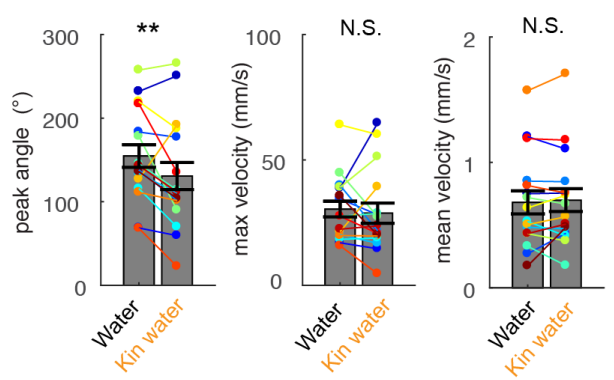

C OXT neurons
$(n=500$ neurons from 16 fish) $\%$ cells in cluster

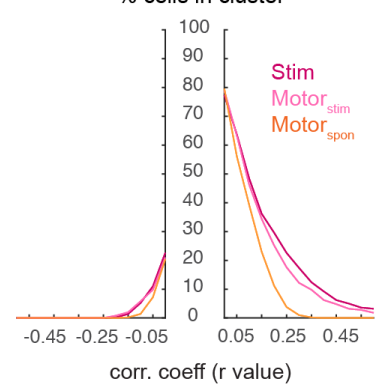

e

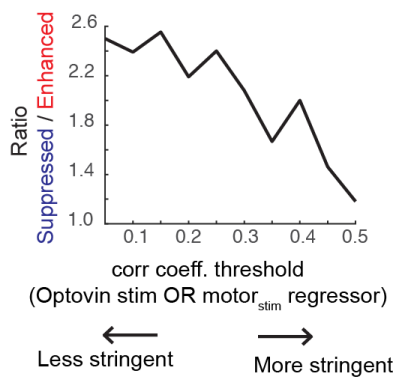

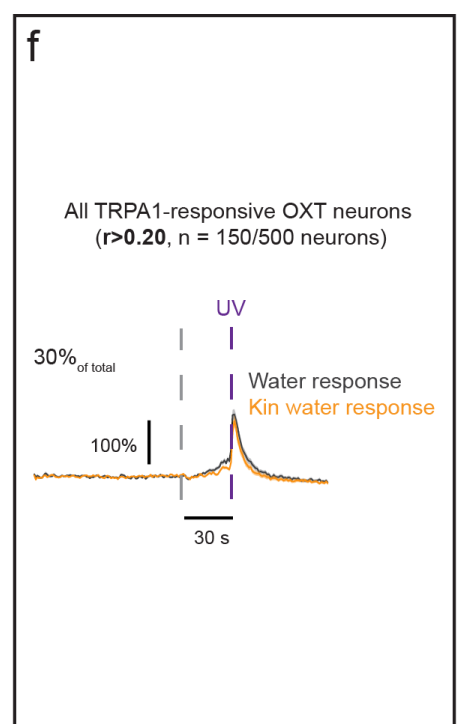
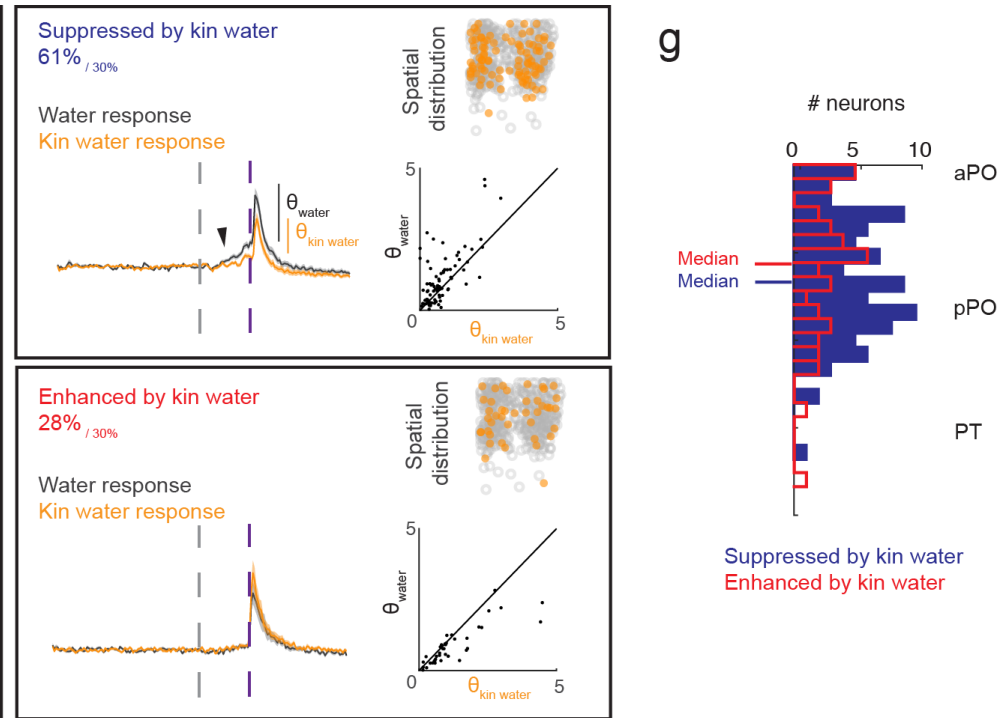

Suppressed by kin water Enhanced by kin water

(a) Top: Schematic showing setup to probe effect of conspecific-conditioned water on TRPA1-induced nocifensive responses. Bottom: Fish were tethered with their nose/mouth and tails freed, and incubated in $15 \mu \mathrm{M}$ Optovin solution. Alternating pulses of water or conspecific water were presented, followed by a 260

Figure 3: Chemical kin cues modulate OXT nociceptive responses and defensive behavior 100 ms pulse of UV light after $30 \mathrm{~s}$ to activate TRPA1 receptors. 
(b) Kin water significantly reduced the mean peak tail angle $\left({ }^{* *} p=0.0081\right)$ of TRPA1-induced tail bends. Maximum and mean velocity
signed-rank test, $\mathrm{n}=16$ fish.

(c) The calcium trace of each OXT neuron was cross-correlated with stimulus regressors or motor regressors either within $5 \mathrm{~s}$ of TRPA1 stimulation ( motor $_{\text {stim }}$ ), or outside of the post-stimulus window $\left(\right.$ (motor $\left._{\text {spon }}\right)$, as in Wee et al $(2019)^{6}$. Panel shows percentage of OXT cells that will be classified as stimulus, motor $_{\text {stim }}$ or motor $_{\text {spon }}$-correlated as a function of Pearson's correlation coefficient threshold ( $r$ value).

(d) Distribution of OXT neurons that are either suppressed (blue) or enhanced (red) by kin water, as a

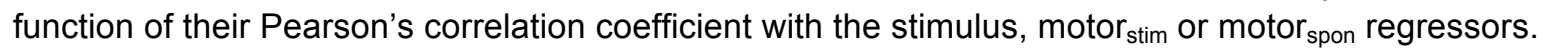
Neurons that show kin water-induced suppression are significantly more correlated to TRPA 1 motor and stimulus regressors ${ }^{* * *} p=7.2 \times 10^{-5}(\mathrm{stim}),{ }^{* * *} p=9.3 \times 10^{-5}$ ( motor $\left._{\text {stim }}\right)$, but not spontaneous movement regressors $(p=0.35)$.

(e) As the threshold for correlation with stim or motor $_{\text {stim }}$ regressors is relaxed (i.e. approaching smaller values), a larger proportion of suppressed rather than activated neurons are observed, suggesting that moderately TRPA1-responsive neurons are most strongly suppressed by kin water. As in Fig. 2, a difference threshold of 0.05 (i.e. $5 \%$ ) was the cutoff for determining if responses were enhanced or suppressed by kin water.

(f) Left panel: Mean stimulus-triggered calcium responses of all TRPA1-responsive neurons in the presence of water (black) or kin-conditioned water (orange), as a function of a medium stim or motor ${ }_{\text {stim }}$ regressor coefficient threshold $(r=0.20)$ used to identify these neurons. See Supplementary Fig. 4 for plots using other $r$-value thresholds. Shading indicates SEM. Gray dashed line $=$ water or kin water delivery, Purple dashed line = UV stimulus onset. Right panels: Mean stimulus-triggered calcium responses for all TRPA1-activated neurons that are suppressed (top) or activated (bottom) by kin water, again for threshold of $r=0.20 . n=151$ neurons (all TRPA1 activated) / 92 neurons (61\%, suppressed by kin water) / 42 neurons ( $28 \%$, activated by kin water) for both control and kin water conditions. Top inset shows the spatial distribution of these neurons. All neurons $(n=500)$ are displayed. Bottom inset compares the magnitude $(\theta)$ of calcium fluorescence $(\Delta f / f)$ change before and after TRPA1 stimulation for neurons that were identified to be suppressed or activated by kin water. Despite the differences in baseline activity that are induced by water flow (see black arrow) the magnitude of calcium fluorescence change post-TRPA1 stimulation is still modulated by kin water.

(g) Distribution along the A-P axis of suppressed and enhanced neurons ( $r>0.20)$.

To further tie nociceptive responses to social modulation of OXT neurons, we examined the effect of conspecific cues on the subset of OXT neurons that respond to TRPA1 stimulation. As we had previously done ${ }^{6}$, each neuron's calcium activity was correlated with stimulus and motor regressors (Fig. 3c). For motor regressors, we further classified responses into those that

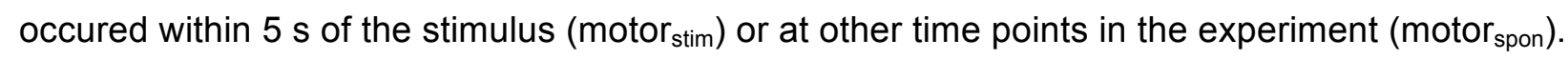
Individual OXT neurons were then subsequently grouped according to whether they displayed suppressed or enhanced TRPA1 responses following kin water presentation. We found that kin 
water-suppressed neurons had significantly higher (right-shifted on the graph shown in Fig. 3d) correlations with TRPA1 stimulus and motor $_{\text {stim }}$ regressors. Indeed, among OXT neurons that had high TRPA1 responsiveness ( $r>0.35 ; 13 \%$ of OXT neurons), a larger fraction of neurons were suppressed (55\%) rather than activated (33\%) by kin water (Supplementary Fig. 4). However, when less stringent criteria was used to define TRPA1 responsiveness (e.g. $r$ $>0.20$, Fig $3 f$ ), the ratio of kin water-suppressed to kin water-activated OXT neurons increased even further (Fig. 3d-e, also see Supplementary Fig. 4), indicating that the bulk of kin watersuppressed OXT neurons are only moderately-responsive to TRPA1. When we included these moderately-responsive OXT neurons in our analysis, we observed a clear divergence from baseline activity before TRPA1 activation, which was induced by water flow alone (Fig. 3f, top right panel), suggesting that these kin water-suppressed OXT neurons had both a water flowinduced and TRPA1-specific response. Thus many kin water-suppressed OXT neurons are not only responsive to TRPA1 stimulation, but also to other potentially aversive stimuli such as water flow.

Notably, kin water was sufficient to reduce responses to both water flow and TRPA1 stimuli (even when accounting for baseline differences; Fig. 3f, top right panel). Further, these kin water-suppressed neurons also tended to be more posteriorly-distributed, consistent with the idea that they correspond to parvocellular, hindbrain-projecting neurons which drive motor output $^{6}$ (Fig. 3g). In contrast, OXT neurons that showed enhanced activation under exposure to kin water were more highly-selective for TRPA1 activation, and did not show apparent responses to water flow (Fig. 3f, bottom right panel). Thus, the reduced nocifensive responses of larval fish observed in the presence of social cues is directly reflected in the suppressed activity of a subset of TRPA1-responsive neurons within the OXT circuit.

OXT neuronal activity suppresses appetite in a conspecific cue-dependent manner

In mammals, social isolation is associated not only with increases in aversive behavior, but also with a reduction in positive-valence behaviors such as feeding ${ }^{22}$. To determine if this is also the case in larval zebrafish, we first made use of an established and quantitative food intake assay that measures gut fluorescence intensity caused by the ingestion of fluorescently-labeled paramecia $^{23-25}$ (Fig. 4a). In this assay, larval fish were food deprived for a period of two hours to increase appetite ${ }^{23-25}$, either in isolation or in the presence of conspecific fish (Fig. 4a).

338 Subsequently, animals maintained under either of these two conditions were presented with 339 large excess of fluorescently labeled paramecia. Notably, fish maintained in isolation consumed 340 significantly less paramecia (i.e. food) than those kept in small groups. Additionally, when group 

size (Fig. 4b). Finally, we observed that water-borne cues from closely related larval fish (kin fish), but not visual cues, were sufficient to rescue the isolation-induced suppression of food intake (Fig. 4c).

\section{FIGURE 4}

a

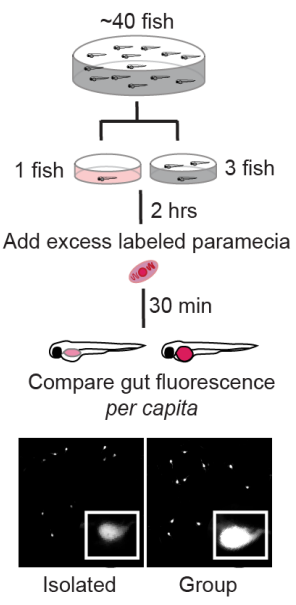

b

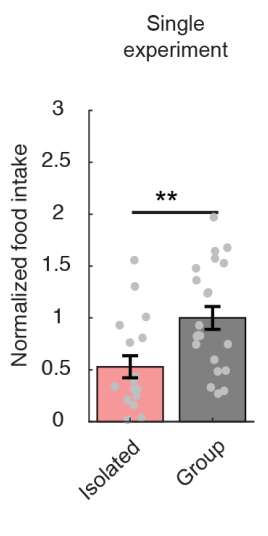

C

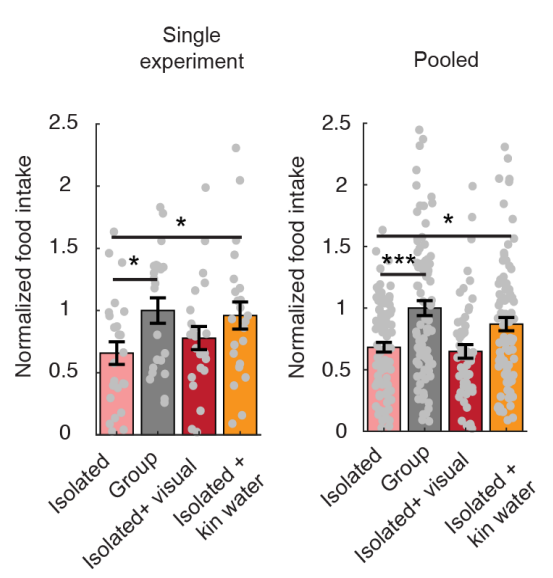

d

\section{7}

348

349

350

351

352

353

354

355

356

357

358 e

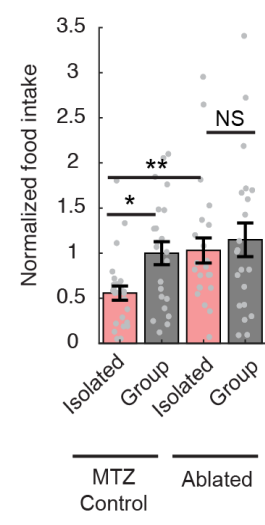

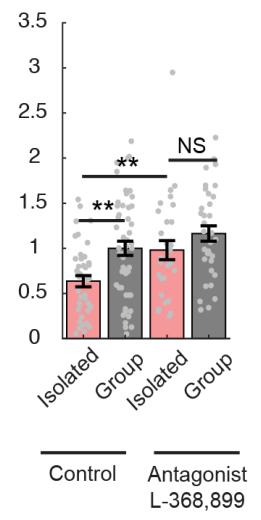

f

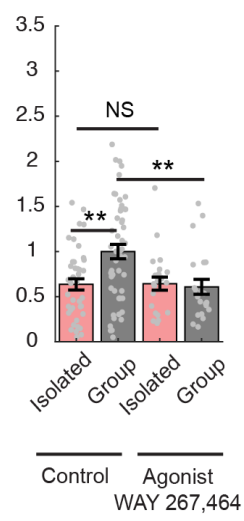

Pooled

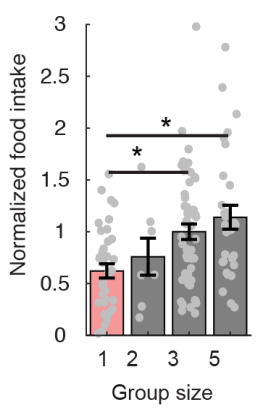

h
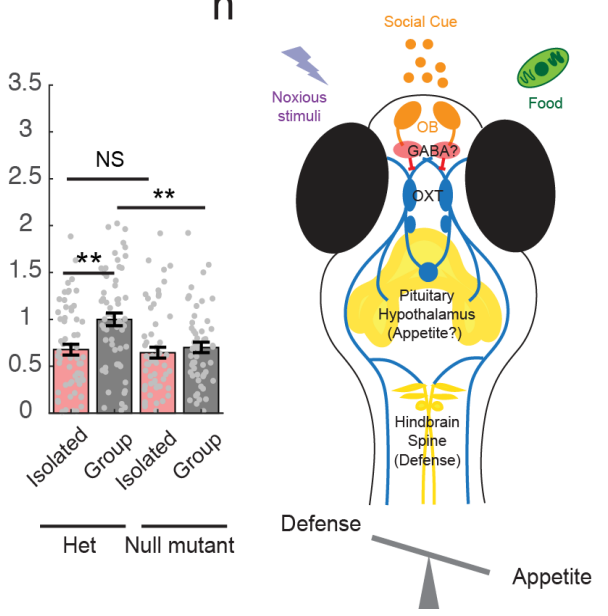

\section{Figure 4: Manipulation of OXT signaling affects appetite in a social-context dependent manner}

(a) Schematic of social behavior experiments. Larval zebrafish were raised in a group up to 7 or $8 \mathrm{dpf}$. They were then either isolated in a small dish or into a small group of 3 fish. After 2 hrs, fluorescentlylabeled paramecia was added for $30 \mathrm{~min}$, following which fish were fixed and subsequently imaged. Example raw fluorescence images are shown, with inset showing higher (100x) magnification image. Integrated gut fluorescence (area $x$ intensity) was used to approximate food intake.

(b) Left: Gut fluorescence quantification from a single experiment demonstrates that isolated fish on average eat less, per capita, than fish in a group of 3 . All gut fluorescence measurements are normalized to the mean food intake of fish in a group $\left({ }^{* *} p=0.0058, n=21 / 17\right.$ fish, two-sided Wilcoxon rank-sum 
test). Right: Normalized food intake (i.e. gut fluorescence normalized to the average gut fluorescence of fish in a group of 3$)$ scales with group size $(n=36,7,52,29$ fish, single asterisks depict $p<0.05$ using one-way ANOVA corrected for multiple comparisons).

(c) Left: Chemical but not visual cues were sufficient to rescue isolation-induced feeding suppression in a single experiment. ${ }^{*} p=0.03$ (single vs group), ${ }^{*} p=0.04$ (single vs kin water), $p=0.17$ (single vs visual), $\mathrm{n}=24 / 24 / 22 / 23$ fish, two-sided Wilcoxon rank-sum test. Right: Average of multiple experiments. $\mathrm{p}=$ ${ }^{* * *} 2.8283 \times 10^{-4}$ (single vs group), * $p=0.02$ (single vs kin water), $p=0.35$ (single vs visual), $n=$ $86 / 86 / 55 / 87$, Wilcoxon rank-sum test.

(d) Cell-specific chemical ablation of OXT neurons specifically rescues effect of social isolation on appetite ( $n=65 / 59 / 57 / 56$ fish). ${ }^{*} p=0.015$ (single vs group), ${ }^{* *} p=0.0017$ (single control vs single ablated), $p=0.76$ (single ablated vs group ablated), two-sided Wilcoxon rank-sum test. Controls are metronidazole (MTZ)-treated, non-transgene-expressing siblings.

(e) Oxytocin antagonist L-368,899 rescues isolation-induced suppression of food intake without significantly changing food intake in a group ( $n=40 / 50 / 29 / 34$ fish). ${ }^{* *} p=0.0022$ (single vs group), ${ }^{* *} p=$ 0.0091 (single control vs single antagonist), $p=0.09$ (single antagonist vs group antagonist), two-sided Wilcoxon rank-sum test.

(f) Oxytocin agonist WAY267,484 suppresses food intake in groups but does not affect food intake in isolated fish ( $n=40 / 50 / 20 / 21$ fish). ${ }^{* *} p=0.0022$ (single vs group), $p=1$ (single control vs single agonist), ${ }^{* *} p=0.0069$ (group control vs group agonist), two-sided Wilcoxon rank-sum test. Control groups for both (e) and (f) are the same sets of fish, split up for better visualization. All data is from multiple sets of experiments and normalized to food intake in a group of 3 fish.

(g) Comparison of food intake of oxt null mutants (oxt-/-) and their heterozygous wild-type siblings (oxt+/-) in isolated and non-isolated contexts ( $n=60 / 59 / 58 / 54$ fish). Single-group differences in food consumption are abolished -- however, food intake was reduced in groups, rather than enhanced in isolated fish. ${ }^{* *} p=0.0011$ (single vs group), $p=0.65$ (single control vs single mutant), ${ }^{* *} p=0.0012$ (group control vs group mutant), two-sided Wilcoxon rank-sum test.

(h) Schematic of our model for how oxytocin neurons can integrate information on social state to control appetite and avoidance behaviors. We posit that social chemical cues are olfactory, and that GABAergic neurons in the forebrain transform OB activation into inhibitory signals. The OXT circuit modulates nocifensive behavior via brainstem premotor neurons ${ }^{6}$. Since it projects extensively into other parts of the hypothalamus, as well as the pituitary gland, these downstream regions may be involved in mediating its effects appetite ${ }^{25}$.

402 (while still in a group of conspecifics) with the prodrug metronidazole (MTZ). This treatment

403 resulted in a loss of $\sim 80 \%$ of nitroreductase-labeled preoptic OXT cells (unablated $=20.3 \pm 1.1$ 
405

406

407

408

409

410

411

412

larvae, or MTZ-treated non-expressing controls, were then separated either into groups of 3 or isolated, and assayed for feeding as described above.

We found that OXT neuron ablation enhanced food intake in isolated animals, while the food intake of fish maintained in a group was unchanged (Fig. 4d). Consistently, addition of an OXT receptor antagonist strongly increased food intake in isolated fish (Fig. 4e), whereas OXT agonists strongly suppressed food intake of fish kept in a group (Fig. 4f). These results indicate that OXT signaling is both necessary and sufficient to mediate the social modulation of food intake observed in socially isolated fish.

Lastly, we compared food intake in OXT homozygous null mutant animals with their heterozygous siblings. As expected, we found that null mutant animals did not modulate their food intake on the basis of social environment, whereas the behavior of their heterozygous siblings was indistinguishable from that of wild type animals. However, mutant animals had low food intake in both the isolated and the group setting (Fig. $4 \mathrm{~g}$ ). Thus, as with acute ablation of OXT neurons, social modulation of feeding was impaired. However, the generally low food intake in these fish might reflect long-term deficits in circuit activity that altered the development or maintenance of neural circuits involved in socially modulated behaviors. In summary, these results demonstrate that, similar to nocifensive behaviors, larval zebrafish OXT neurons are necessary and sufficient for social state modulation of appetite.

\section{DISCUSSION}

Larval zebrafish are generally not thought to exhibit robust social interactions, aside from simple behaviors such as rudimentary avoidance of other larvae ${ }^{26}$. However, we found that even brief $(2 \mathrm{hr})$ social isolation results in distinct neural signatures that correlate with aversive and nociceptive brain circuits, including activity in the preoptic area, posterior tuberculum, caudal hypothalamus and, notably, populations of oxytocin (OXT)-positive neurons. This is consistent with the results of a recent study (Tunbak et al, preprint online ${ }^{27}$ ) that described increased activity in the preoptic area as a result of long-term social isolation in older (juvenile, $21 \mathrm{dpf}$ ) zebrafish. Thus, in an intriguing parallel to humans ${ }^{28}$, we note that pain and social isolation exhibit a shared neural signature in the larval zebrafish brain that is likely to extend also to older animals in this species.

Extending the analysis of the OXT-positive population revealed that these neurons are functionally diverse and modulate distinct behavioral outputs, including feeding and nocifensive escape responses (see also Wee et al, $2019^{6}$ ). We thus propose a circuit mechanism by which the larval zebrafish integrates conspecific social cues into the modulation of defensive and 
appetitive behaviors (Fig. 3h). Our data suggest that OXT neurons (and likely other preoptic populations; Supplementary Fig. 1) encode a range of chemical social information via olfactory inputs (Supplementary Fig. 2), which then becomes the basis for the modulation of nocifensive and appetitive behavioral outputs. Indeed, our data reveal that the presence of conspecifics, and specifically conspecific chemical cues, significantly increases appetite and reduces nociceptive responses (Fig. $3 \& 4$ ). At the circuit level, OXT neurons displayed a variety of responses to these chemical social cues, which could thus uniquely signal the presence of adult fish (known predators), genetically-related animals (larval kin), or distantly-related (larval nonkin) conspecifics. Importantly, we show that kin cues induced the most widespread inhibition of OXT neurons (Fig. 2), while the same OXT neurons can be either excited or inhibited by other cues, suggesting multimodal and valence-specific tuning. Notably, conspecific cues increase the activity of a small subset of OXT neurons that appear to be more sensory in nature suggesting that these could drive anxiolytic or anti-nociceptive effects, consistent with traditional views of OXT function ${ }^{3}$.

In humans and other mammals, it is known that social cues, including odors, can attenuate aversive experience and behaviors, a phenomenon known as "social buffering"20,21. Social facilitation of appetite has also been observed in many species ${ }^{29}$, and is likely evolutionarily adaptive. In general, an isolated animal needs to shift its priorities from foraging to vigilance or escape, since it may be more susceptible to the risk of predation. Accordingly, adult zebrafish display isolation stress in a group size-dependent manner ${ }^{30}$. We had previously shown that OXT neurons respond to a range of aversive, particularly noxious stimuli, and are sufficient to drive motor responses by acting on brainstem targets ${ }^{6}$. By demonstrating that chemical social cues converge on this circuit, and that kin cues, in particular, predominantly diminish the activity of TRPA1-responsive, parvocellular OXT neurons, we provide a potential mechanistic understanding of how the OXT circuit can mediate the phenomenon of "social buffering" in a vertebrate organism ${ }^{31}$.

Furthermore, our demonstration of a suppressive effect of OXT on appetite corroborates a series of mammalian research findings: 1) The insatiable appetite and morbid obesity observed in Prader Willi Syndrome is likely due to impaired OXT signaling ${ }^{32,33}$; 2) Acute 468 inhibition of paraventricular OXT neurons can promote food intake ${ }^{34}$; 3) Lesions of the PVN, as 469 well as mutations that affected OXT neuron development, have been shown to cause 470 hyperphagia and obesity ${ }^{35,36}$, and; 4) Direct administration of OXT has been shown to reduce 471 feeding ${ }^{4,5}$. At the same time, our data suggests that the role of OXT in feeding may be more 472 complex than previously appreciated. Notably, while OXT homozygous mutants do not show 
social state-dependent modulation of appetite, they also eat less than their heterozygous siblings, implying that a complete absence of OXT may be detrimental towards feeding.

Our data further highlight the profound influence of social context on OXT's appetitesuppressing effects, which may very well generalize to mammals. For example, a recent study found that inhibiting OXT signaling enhances sugar intake in a socially dominant mouse regardless of their social context, whereas in subordinate mice, such inhibition only enhanced appetite when cues from the dominant mouse were not present ${ }^{37}$. Our results also complement the observations of strong interactions between social and feeding circuits across evolution ${ }^{38}$, and reinforce a role for OXT in prioritizing various motivated behaviors ${ }^{4}$. However, effects of OXT in larval zebrafish may occur as part of a coordinated response to both social isolation and noxious contexts, rather than reproduction or parental care.

We do note some important distinctions between our findings and the canonical view of OXT function as suggested by mammalian studies, the most significant of which is that the larval zebrafish OXT neurons show widespread activation by social isolation, rather than by cues indicating the presence of conspecifics ${ }^{2,39}$. We propose three possible reasons for these apparent differences: first, representations within both the mammalian and zebrafish OXT population are diverse and thus the observed activity patterns in zebrafish may reflect those of specific subpopulations of mammalian OXT cells (indeed, some zebrafish OXT neurons are activated by conspecific cues, and some mammalian OXT cells are inhibited by conspecific cues $^{39}$ ); second, OXT response properties may have changed over the course of evolution, as more sophisticated social functions were derived. Third, there is a possibility that OXT neuron response properties might reverse over the course of development, since adult and kin odor generate opposite activity signatures; however, since the enhancement of preoptic area activation appears to persist at least till juvenile stages (Tunbak et al, preprint online ${ }^{27}$ ), when social preference behaviours have developed, any such reversal would have to happen closer to adulthood. Overall, our results may provide a broader and more intricate perspective of OXT's social function in vertebrate animals.

Furthermore, given that noxious stimuli and social isolation both activate the OXT population, an enhancement of OXT signaling may in fact represent a negative valence state in larval zebrafish, rather than the rewarding experience it is generally associated with.

503 Interestingly, recent studies in mammalian models have demonstrated that OXT neurons can 504 also be negatively reinforcing, and promote fear, stress and anxiety in some situations ${ }^{1,40}$. Thus, 505 this study could provide an evolutionary perspective on the ancient functions of this highlyconserved peptide and how they relate to our current understanding of them in mammals. 
In conclusion, our study demonstrates how organizing principles and circuit implementation strategies underlying social behavior can be elucidated by probing social context-dependent behaviors in a small and optically accessible model organism. More broadly, our dissection of the larval zebrafish OXT circuit provides an entrypoint into understanding how neuromodulatory systems represent behavioral states such as social isolation, hunger, and acute nociception, on multiple timescales, and how these representations are then used to modulate behavioral output in a flexible and context-dependent manner.

\section{METHODS}

Fish husbandry and transgenic lines

518 Larvae and adults were raised in facility water and maintained on a 14:10 hr light:dark cycle at $28^{\circ} \mathrm{C}$. All protocols and procedures involving zebrafish were approved by the Harvard University/Faculty of Arts \& Sciences Standing Committee on the Use of Animals in Research and Teaching (IACUC). Fish were raised at a density of $\sim 40$ fish per dish and fed from $5 \mathrm{dpf}$ till the day of the experiment. Behavioral experiments were carried out mostly on fish of the WIK background, although other genotypes (e.g. $A B$, or mit1fa-/- (nacre) in the AB background) were also utilized and showed similar behavioral results. mit1fa-/- (nacre) in the AB background, along with additional transgenes described below, were also used for calcium imaging and MAP-mapping experiments. Transgenic lines Tg(oxt:GFP ${ }^{41}, \operatorname{Tg}(U A S: G C a M P 6 s){ }^{42}$, Tg(UAS:nsfbCherry) ${ }^{43}, T g(H u C: G C a M P 6 s)^{44}, T g$ (oxt:Gal4) and oxytocin mutants ${ }^{6}$ were previously published.

\section{MAP-mapping}

7-8 dpf larvae, that had been continuously fed with an excess of paramecia since $5 \mathrm{dpf}$, were either isolated or split into small groups, using $35 \mathrm{~mm}$ dishes filled with $3 \mathrm{ml}$ embryo water. For groups of 10 , a larger $(10 \mathrm{~cm})$ dish was used to prevent overcrowding. Paramecia was present within each dish to ensure that the fish were well-fed and had ample stimulation. After $2 \mathrm{hrs}$, larvae were quickly funneled through a sieve, which was then quickly dropped into $4 \%$ paraformaldehyde, immunostained, imaged and analyzed as described in Randlett et al. $(2015)^{11}$.

\section{Exposure to sensory cues for high-resolution pERK experiments} continuously fed with an excess of paramecia from $5 \mathrm{dpf}$ were transferred into a new $10 \mathrm{~cm}$ petri dish that did not contain any paramecia, at a concentration of 2 fish per ml. After a $2 \mathrm{hr}$ incubation, a syringe with an attached $0.45 \mu \mathrm{m}$ filter was used to very gently suck out the conditioned water, with great care taken not to disturb or stress the fish in the process.

\section{7-8 dpf larvae, that had been continuously fed with an excess of paramecia since $5 \mathrm{dpf}$, were} either isolated or split into small groups, using $35 \mathrm{~mm}$ dishes filled with $3 \mathrm{ml}$ embryo water. 
Paramecia was present within each dish to ensure that the fish were well-fed and had ample stimulation. $700 \mu$ of the filtered conspecific-conditioned water was added to each $35 \mathrm{~mm}$ dish ( 1:5 dilution), 30 min before fixation, and embryo water was correspondingly added to controls. For providing visual access to conspecifics, the $35 \mathrm{~mm}$ dishes containing single larvae were inserted into larger $(55 \mathrm{~mm})$ dishes containing $\sim 5$ larvae that thus be surrounding but unable to interact with the single larva. After 2 hrs, larvae were quickly funneled through a sieve, which was then quickly dropped into 4\% paraformaldehyde, dissected in PBS and immunostained as described in Wee et al, $2019^{6}$.

\section{High resolution $p E R K$ analysis}

For quantification of $\mathrm{pERK/tERK}$ ratios over individual OXT neurons, $\mathrm{pERK}$ experiments were performed on dissected $T g(o x t: G F P)$ brains. Cellular-resolution imaging of dissected brains was obtained using the Zeiss (LSM 700 and LSM 880) or Olympus (FVB1000MPE) confocal microscopes. pERK/tERK intensities of individual GFP-positive neurons were measured using Image $J$ and quantified using MATLAB as reported in Wee et al, $2019^{6}$. Analysis code is also available on www.github.com/carolinewee

\section{Social feeding experiments}

For experiments in which feeding was assessed, larvae that had been continuously fed with an excess of paramecia from $5 \mathrm{dpf}$ were either isolated or placed in groups of 3 , in $35 \mathrm{~mm}$ dishes ( $3 \mathrm{ml}$ embryo water), in the absence of food. After 2 hours, fluorescent-labeled paramecia was added followed by a quick fixation after $30 \mathrm{~min}$ (full protocol is described in Wee et al, eLife, $\left.2019^{25}\right)$. Sensory cues were generated and presented as described above for pERK experiments. Fixed larvae were subsequently distributed into 96-well flat-bottom dishes and imaged using the AxioZoom V16 (Zeiss) and analyzed using Fiji software (3D Objects Counter, custom software also available on www.github.com/carolinewee)

\section{Calcium imaging and olfactory stimulation} imaging experiments. They were embedded in the center of a $55 \mathrm{~cm}$ dish in $1.5 \%$ agarose with their tails and noses freed.

Kin or non-kin conditioned water (at a concentration of $1 \mathrm{fish} / \mathrm{ml}$ ) were generated as described above. In these calcium imaging experiments, we used WIK fish as non-kin fish, since the Tg(oxt:Gal4;UAS:GCaMP6s) fish we imaged were of the AB genetic background. For adult water, 5 adult kin (from parent tank of larvae) were used to condition $500 \mathrm{ml}$ of water (1 adult fish/100 ml) for $2 \mathrm{hrs}$, and also subsequently filtered. Kin and non-kin fish used to condition water were raised apart from experimental fish from $3 \mathrm{dpf}$, to dissociate genetic from familiarity efects.

Alternating olfactory stimuli were delivered using a custom-built syringe pump system controlled by custom Labview software. At specified time intervals, $300 \mu \mathrm{l}$ of each cue ( $30 \mu \mathrm{l} / \mathrm{second})$ was delivered using a zero-dead-volume multi-channel perfusion pencil (AutoMate Scientific). Embryo water was also constantly circulated through the dish using a peristaltic pump (Harvard 
Apparatus). The interstimulus interval (ISI) was 2 minutes for olfactory bulb imaging and 5 minutes for OXT neuron imaging. The longer ISI for OXT neuron imaging was implemented to reduce desensitization and ensure that activity truly returned to baseline before presenting the subsequent stimulus. In order to reduce experiment time and avert the possibility of OXT neuron desensitization, we also never compared responses to more than 2 cues (e.g. either kin vs non-kin, or kin vs adult, but not all 3 stimuli).

Calcium imaging and behavioral monitoring with TRPA1 stimulation was performed as previously reported ${ }^{6}$ on 8-10 dpf larvae, with a number of core differences: 1) $15 \mu \mathrm{M}$ instead of $25 \mu \mathrm{M}$ Optovin (Tocris Bioscienes) was used, to reduce background signals (see below) 2) nostrils are exposed to allow for olfactory stimulation 3 ) the same UV stimulus intensity used throughout the experiment, and only 4 stimulations were presented (alternating kin and water). Since, in addition to the tail, the nose was exposed in this current paradigm, Optovin, a colored solution, was rapidly absorbed into the fish's brain and caused a linear increase in background signal (i.e. even in non-GCaMP-labeled tissue) over time. Post-hoc subtraction of this background signal from OXT GCaMP signals restored a flat baseline, allowing us to extract meaningful calcium signals.

\section{Data analysis}

All calcium imaging data was analyzed using custom ImageJ and MATLAB software. The general protocol for analysis was: 1) Image registration to correct for motion artifacts using the TurboReg ${ }^{45}$ plugin in ImageJ; 2) Extraction of fluorescence signals from both channels using manually-segmented ROIs in MATLAB or on a voxel-by-voxel basis 3) Calculation of $\Delta f / f$ signals from raw traces and alignment to tail traces, as needed, in MATLAB.

For fish expressing $\mathrm{Tg}$ (UAS:GCaMP6s) exclusively in oxt-expressing neurons, ROIs were drawn over all visible cells in a maximum projection image for each plane and raw fluorescence traces were extracted as the mean pixel value within the ROI. Other analysis was done on a voxel-by-voxel basis, rather than using cell segmentation, which would exclude responses from neuropil that are abundant in the OB and forebrain. $\Delta \mathrm{f} / \mathrm{f}$ values were calculated from raw traces using the average fluorescence over the time period before the first stimulus as the baseline to which all traces were normalized.

Behavior, stimulation and calcium imaging timestamps were aligned and used to extract stimulus-triggered averages as well as to generate motor and stimulus regressors to correlate with calcium activity. The regressors were convolved with a GCaMP6s kernel based on its measured response delay (0.48 s) and decay time (3s, based on Chen et al. (2013) and crosscorrelated with calcium traces that had been smoothed with a 3-frame zero phase filter. In order to determine if an OXT neuron was activated or suppressed, we averaged the calcium signal over a 60s interval post-stimulus for each stimulus type. If the difference between the two integrated calcium signal during olfactory and water stimulation was more than 0.05 , we classified the neurons as being either activated or suppressed by the cue depending on the sign. Although the threshold of 0.05 is arbitrary, we show that across a range of thresholds, the relationship between the proportions of suppressed and enhanced neurons remains consistent 
for each type of stimulus. In addition, the first stimulus was always dropped from analysis, in order to account for possible effects of initial startle.

Since there was no difference in kin water responses between younger ( $8 \mathrm{dpf}(\mathrm{n}=7 \mathrm{fish}): 35 \%$ suppressed, $10 \%$ enhanced) and old ( $11 \mathrm{dpf}(\mathrm{n}=7 \mathrm{fish}): 37 \%$ suppressed, $10 \%$ enhanced) fish, we pooled data across all the ages. Fish were isolated for between $30 \mathrm{~min}$ to $4 \mathrm{hrs}$ before imaging, though the bulk of experiments were performed with 1.5-2.5 hr isolation (note that time of isolation $=$ time of embedding). We did not observe any consistent changes in kin water suppression with isolation times, thus any differences observed are likely due to random variation (1 hr or less isolation ( $\mathrm{n}=5$ ): $50 \%$ suppressed, $13 \%$ enhanced; $1.5-2.5 \mathrm{hrs}$ isolation $(n=19): 33 \%$ suppressed, $13 \%$ enhanced, $3-4$ hrs isolation $(n=7): 40 \%$ suppressed, $5 \%$ enhanced).

Free-swimming TRPA1 stimulation

Fish were singly placed into a $20.6 \mathrm{~mm}$ cut-out agarose circular mold illuminated by three quad blue LEDs (Luxeon Star, $470 \mathrm{~nm}$ ). To probe the effect of conspecific cues, DMSO or Optovin solutions were generated either in embryo water or kin-conditioned water. Following 5 min of habituation in DMSO (effectively also the isolation period), fish were stimulated once every $30 \mathrm{~s}$ with a $100 \mathrm{~ms}$ pulse of blue light. The DMSO solution was then exchanged with Optovin, and the same protocol repeated. Behavior was recorded at $200 \mathrm{fps}$ (Pike F-032, Allied Vision) and analyzed using custom Python software.

\section{Statistics}

All error bars show mean \pm SEM over fish. Significance was reported as follows: * $p<0.05$, ${ }^{* *} p<0.01,{ }^{* * *} p<0.001$. Significance was determined using the Wilcoxon signed-rank test for paired data and the Wilcoxon rank-sum test for independent samples. One-sided tests were used in cases where there was a clear hypothesis for the direction of effect. One-way ANOVA with Bonferroni Correction was used in cases where there were multiple comparisons. Wilcoxon signed rank test was used in Fig. 1a for comparing the distribution of normalized OXT ROI signals across different behavioral stimuli to the null hypothesis of median 1.

\section{DATA AVAILABILITY}

All data, code (hardware control and analysis) and resources (transgenic lines/mutants generated) will be made available by the corresponding author upon request.

\section{CODE AVAILABILITY}

Live versions of the analysis code are maintained at www.github.com/carolinewee.

\section{ACKNOWLEDGEMENTS}

We are grateful to Adam Douglass (Univ. of Utah) for continued support, advice, and comments. We thank the CBS imaging facility, Harvard Center for Biological Imaging, and the NorthWest Undergraduate Teaching Laboratories at Harvard for the successful completion of many experiments. J. Miller, K. Hurley and B. Hughes provided invaluable fish care. This work was supported by U01-NS090449 (FE), R24-NS086601 (FE), U19-NS104653 (FE and SK) and 
Simons Foundation grant SCGB 325207 (FE). CLW was supported by the National Science Scholarship from the Agency for Science, Technology and Research (A*STAR), Singapore.

\section{AUTHOR CONTRIBUTIONS}

C.L.W., E.S., and S.K. originally conceived of the project, which was then developed into its final form with M.N and F.E.

684 S.K. \& F.E. supervised the project. C.L.W., E.S., and M.N. designed and performed most of the experiments, and analyzed most of the data. M.N. also developed hardware and software for calcium imaging and behavioral experiments, and analyzed the free-swimming behavioral data. S.W. performed feeding experiments and analyzed data. C.L.W., S.K., and F.E. wrote the manuscript with contribution from all other authors.

\section{COMPETING INTERESTS}

The authors declare no competing interests.

1. Rash, J. A., Aguirre-Camacho, A. \& Campbell, T. S. Oxytocin and pain: a systematic review and synthesis of findings. Clin. J. Pain 30, 453-462 (2014).

2. Lieberwirth, C. \& Wang, Z. Social bonding: regulation by neuropeptides. Front. Neurosci. 8, 171 (2014).

3. Jezova, D., Skultetyova, I., Tokarev, D. I., Bakos, P. \& Vigas, M. Vasopressin and Oxytocin in Stress. Ann. N. Y. Acad. Sci. 771, 192-203 (1995).

4. Leng, G., Onaka, T., Caquineau, C., Sabatier, N., Tobin, V. A. \& Takayanagi, Y. Oxytocin and appetite. Prog. Brain Res. 170, 137-151 (2008).

5. Sabatier, N., Leng, G. \& Menzies, J. Oxytocin, feeding, and satiety. Front. Endocrinol. 4, 35 (2013).

6. Wee, C. L., Nikitchenko, M., Wang, W.-C., Luks-Morgan, S. J., Song, E., Gagnon, J. A., 
avoidance. bioRxiv 745075 (2019). doi:10.1101/745075

8. Herget, U., Gutierrez-Triana, J. A., Salazar Thula, O., Knerr, B. \& Ryu, S. Single-Cell Reconstruction of Oxytocinergic Neurons Reveals Separate Hypophysiotropic and Encephalotropic Subtypes in Larval Zebrafish. eneuro 4, ENEURO.0278-16.2016 (2017).

9. Stoop, R. Neuromodulation by oxytocin and vasopressin. Neuron 76, 142-159 (2012).

10. Knobloch, H. S. \& Grinevich, V. Evolution of oxytocin pathways in the brain of vertebrates. Front. Behav. Neurosci. 8, 31 (2014).

11. Randlett, O., Wee, C. L., Naumann, E. A., Nnaemeka, O., Schoppik, D., Fitzgerald, J. E., Portugues, R., Lacoste, A. M. B., Riegler, C., Engert, F. \& Schier, A. F. Whole-brain activity mapping onto a zebrafish brain atlas. Nat. Methods 12, 1039-1046 (2015).

12. Wircer, E., Blechman, J., Borodovsky, N., Tsoory, M., Nunes, A. R., Oliveira, R. F. \& Levkowitz, G. Homeodomain protein Otp affects developmental neuropeptide switching in oxytocin neurons associated with a long-term effect on social behavior. Elife 6, (2017).

13. Herget, U., Wolf, A., Wullimann, M. F. \& Ryu, S. Molecular neuroanatomy and chemoarchitecture of the neurosecretory preoptic-hypothalamic area in zebrafish larvae. J. Comp. Neurol. 522, 1542-1564 (2014).

14. Gerlach, G., Hodgins-Davis, A., Avolio, C. \& Schunter, C. Kin recognition in zebrafish: a 24hour window for olfactory imprinting. Proc. Biol. Sci. 275, 2165-2170 (2008).

15. Biechl, D., Tietje, K., Gerlach, G. \& Wullimann, M. F. Crypt cells are involved in kin recognition in larval zebrafish. Sci. Rep. 6, 24590 (2016).

16. Rauch, G.-J., Granato, M. \& Haffter, P. A polymorphic zebrafish line for genetic mapping using SSLPs on high-percentage agarose gels. Tech. Tips Online 2, 148-150 (1997). isotocin neurons in the brain of rainbow trout: central and neurohypophysial projections of single neurons. Neuroscience 124, 973-984 (2004).

18. Swanson, L. W. \& Sawchenko, P. E. Paraventricular nucleus: a site for the integration of 
neuroendocrine and autonomic mechanisms. Neuroendocrinology 31, 410-417 (1980).

19. Sawchenko, P. E. \& Swanson, L. W. Immunohistochemical identification of neurons in the paraventricular nucleus of the hypothalamus that project to the medulla or to the spinal cord in the rat. J. Comp. Neurol. 205, 260-272 (1982).

20. Kikusui, T., Winslow, J. T. \& Mori, Y. Social buffering: relief from stress and anxiety. Philos. Trans. R. Soc. Lond. B Biol. Sci. 361, 2215-2228 (2006).

21. Kiyokawa, Y. Social Odors: Alarm Pheromones and Social Buffering. Curr. Top. Behav. Neurosci. (2015). doi:10.1007/7854_2015_406

22. Bazhan, N. \& Zelena, D. Food-intake regulation during stress by the hypothalamo-pituitaryadrenal axis. Brain Res. Bull. 95, 46-53 (2013).

23. Shimada, Y., Hirano, M., Nishimura, Y. \& Tanaka, T. A high-throughput fluorescence-based assay system for appetite-regulating gene and drug screening. PLoS One 7, e52549

24. Jordi, J., Guggiana-Nilo, D., Soucy, E., Song, E. Y., Wee, C. L. \& Engert, F. A highthroughput assay for quantifying appetite and digestive dynamics. Am. J. Physiol. Regul. Integr. Comp. Physiol. ajpregu.00225.2015 (2015). doi:10.1152/ajpregu.00225.2015

25. Wee, C. L., Song, E. Y., Johnson, R. E., Ailani, D., Randlett, O., Kim, J.-Y., Nikitchenko, M., Bahl, A., Yang, C.-T., Ahrens, M. B., Kawakami, K., Engert, F. \& Kunes, S. A bidirectional network for appetite control in larval zebrafish. Elife 8, (2019).

26. Dreosti, E., Lopes, G., Kampff, A. R. \& Wilson, S. W. Development of social behavior in young zebrafish. Front. Neural Circuits 9, 39 (2015).

27. Tunbak, H., Vazquez-Pradam, M., Ryan, T., Kampff, A. R. \& Dreosti, E. The lonely fish is not a loner fish: whole-brain mapping reveals abnormal activity in socially isolated zebrafish. bioRxiv 2020.01.22.915520 (2020). doi:10.1101/2020.01.22.915520 of Social Exclusion. Science 302, 290-292 (2003). 
29. Higgs, S. \& Thomas, J. Social influences on eating. Current Opinion in Behavioral Sciences 9, 1-6 (2016).

30. White, L. J., Thomson, J. S., Pounder, K. C., Coleman, R. C. \& Sneddon, L. U. The impact of social context on behaviour and the recovery from welfare challenges in zebrafish, Danio rerio. Anim. Behav. 132, 189-199 (2017).

31. Smith, A. S. \& Wang, Z. Hypothalamic oxytocin mediates social buffering of the stress response. Biol. Psychiatry 76, 281-288 (2014).

32. Swaab, D. F. Neuropeptides in hypothalamic neuronal disorders. Int. Rev. Cytol. 240, 305375 (2004).

33. Francis, S. M., Sagar, A., Levin-Decanini, T., Liu, W., Carter, C. S. \& Jacob, S. Oxytocin and vasopressin systems in genetic syndromes and neurodevelopmental disorders. Brain Res. 1580, 199-218 (2014).

34. Atasoy, D., Betley, J. N., Su, H. H. \& Sternson, S. M. Deconstruction of a neural circuit for hunger. Nature 488, 172-177 (2012).

35. Xi, D., Gandhi, N., Lai, M. \& Kublaoui, B. M. Ablation of Sim1 neurons causes obesity through hyperphagia and reduced energy expenditure. PLoS One 7, e36453 (2012).

36. Tolson, K. P., Gemelli, T., Meyer, D., Yazdani, U., Kozlitina, J. \& Zinn, A. R. Inducible neuronal inactivation of Sim1 in adult mice causes hyperphagic obesity. Endocrinology 155,

37. Olszewski, P. K., Allen, K. \& Levine, A. S. Effect of oxytocin receptor blockade on appetite for sugar is modified by social context. Appetite 86, 81-87 (2015).

38. Fischer, E. K. \& O'Connell, L. A. Modification of feeding circuits in the evolution of social behavior. J. Exp. Biol. 220, 92-102 (2017).

39. Resendez, S. L., Namboodiri, V. M. K., Otis, J. M., Eckman, L. E. H., RodriguezRomaguera, J., Ung, R. L., Basiri, M. L., Kosyk, O., Rossi, M. A., Dichter, G. S. \& Stuber, 
of the hypothalamus to promote social behavior in male mice. J. Neurosci. (2020). doi:10.1523/JNEUROSCI.1515-18.2020

40. Grillon, C., Krimsky, M., Charney, D. R., Vytal, K., Ernst, M. \& Cornwell, B. Oxytocin increases anxiety to unpredictable threat. Mol. Psychiatry 18, 958-960 (2013).

41. Coffey, C. M., Solleveld, P. a., Fang, J., Roberts, A. K., Hong, S.-K., Dawid, I. B., Laverriere, C. E. \& Glasgow, E. Novel oxytocin gene expression in the hindbrain is induced by alcohol exposure: transgenic zebrafish enable visualization of sensitive neurons. PLoS One 8, e53991 (2013).

42. Muto, A., Lal, P., Ailani, D., Abe, G., Itoh, M. \& Kawakami, K. Activation of the hypothalamic feeding centre upon visual prey detection. Nat. Commun. 8, 15029 (2017).

43. Davison, J. M., Akitake, C. M., Goll, M. G., Rhee, J. M., Gosse, N., Baier, H., Halpern, M. E., Leach, S. D. \& Parsons, M. J. Transactivation from Gal4-VP16 transgenic insertions for tissue-specific cell labeling and ablation in zebrafish. Dev. Biol. 304, 811-824 (2007). zebrafish. Nat. Methods 14, 1107-1114 (2017). 